\title{
Mitral valve endocarditis - how to treat a non-cooperating patient
}

\author{
(1)Andreja Čleković- \\ Kovačić*, \\ DRenata Ivanac \\ Janković, \\ Olvana Petrović Juren, \\ (1) Vlasta Soukup \\ Podravec
}

Bjelovar General Hospital, Bjelovar, Croatia
RECEIVED:

February 28, 2019

ACCEPTED:

March 24, 2019

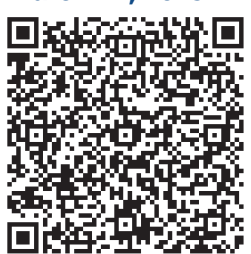

$\square$ Cardiologia Croatica 2019;14(3-4):50.
KEYWORDS: mitral valve endocarditis, Coagulase-negative Staphylococci, end stage renal disease, transthoracic echocardiogram, transesophageal echocardiography.

CITATION: Cardiol Croat. 2019;14(3-4):50. | https://doi.org/10.15836/ccar2019.50

*ADDRESS FOR CORRESPONDENCE: Andreja Čleković-Kovačić, Opća bolnica Bjelovar, A. Mihanovića 8, HR-43000 Bjelovar, Croatia. / Phone: +385-43-279-181 / E-mail: kovacicandreja1@gmail.com

ORCID: Andreja Čleković-Kovačić, https://orcid.org/0000-0002-4532-3597 • Renata Ivanac Janković, https://orcid.org/0000-0003-4949-3953 Ivana Petrović Juren, https://orcid.org/0000-0002-2793-3455 • Vlasta Soukup Podravec, https://orcid.org/0000-0002-4605-0068

\section{|I||||||||||||||||||||||||||||||||||||||||||||||||||||||||||||||||||||||||||||||||||||||||||||||||||||||||||||||||||||||||||||||}

Introduction: Coagulase-negative staphylococci (CoNS) are gram-positive cocci which are common inhabitants of the skin. Infections are usually associated with intravascular catheters, artificial heart valves, vascular grafts and prosthetic joints. ${ }^{1,2}$ We herein present the case of 44 -year-old female who presented with an aggressive form of infective endocarditis involving the mitral valve following a central venous catheter due to infection with CoNS

Case report: 44-year-old female patient presented with end stage renal disease of unknown origin has been hospitalized for the first day of hemodialysis. At the arrival she was febrile (38 degrees Celsius), with chills and extremely messy place of the temporary non-tunneled jugular catheter insertion. Laboratory findings showed highly elevated inflammatory parameters and chest X-ray was normal. Blood cultures were taken, and because of basic suspicion of catheter-related infections, it was extracted and directed to a microbiological analysis which pointed coagulase negative Staphylococci infection vancomycin-sensitive. Meanwhile, transthoracic and transesophageal echocardiography was performed and vegetation on mitral valve, measured $8 \times 12 \mathrm{~mm}$ was detected. Despite antimicrobial therapy, patient was febrile, and infection was uncontrolled, and vegetation was of impressive size. CT scan of abdomen and brain were negative to abscess or embolization, so tertiary center was contacted and further observation at the clinic was indicated, but patient refused it. Fortunately, in the control interval the vegetation began to decrease (up to $8 \times 9 \mathrm{~mm}$ ), the patient became afebrile and insisted on leaving the hospital. Therapy continued during regular hemodialysis up to a complete of 6 weeks. After treatment, control transesophageal echocardiography shows residual changes of mitral valve vegetation accompanied smaller mitral regurgitation, and hemodialysis flow properly.

Conclusion: A vegetation size larger than $10 \mathrm{~mm}$ accompanied with uncontrolled infection in the absence of developed complications is cutoff for consideration of operative treatment. We rarely count on patient noncompliance, but these patients are special challenge for treatment both in reference centers and in county hospitals.
LITERATURE IIIIIIIIIIIIIIIIIIIIIIIIIIIIIIIIIIIIIIIIIIIIIIIIIIIIIIIIIIIIIIIIIIIIIIIIIIIIIIIIIIIIIIIIIIIIIIIIIIIIIIIIII

1. Habib G, Lancellotti P, Antunes MJ, Bongiorni MG, Casalta JP. Del Zotti F, et al: ESC Scientific Document Group. 2015 ESC Guidelines for the management of infective endocarditis: The Task Force for the Management of Infective Endocarditis of the European Society of Cardiology (ESC). Endorsed by: European Association for Cardio-Thoracic Surgery (EACTS), the European Association of Nuclear Medicine (EANM). Eur Heart J. 2015 Nov 21;36(44):3075-3128. https://doi.org/10.1093/eurheartj/ehv319

2. Tuffariello JAM, Lowy FD. Infection due to coagulase-negative staphylococci: Treatment. Available from: http://www.uptodate.com (February 28, 2019). 